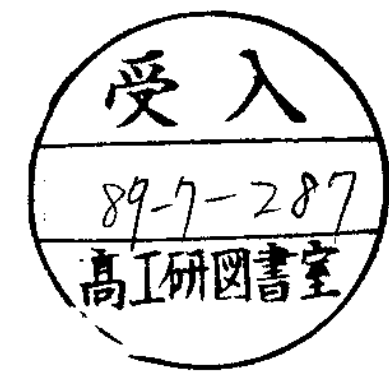

IC/89/40

CERN-TH.5360/89

\title{
LANGEVIN FORMULATION OF QUANTUM DYNAMICS
}

\author{
Marco Roncadelli \\ International Centre for Theoretical Physics, Trieste, Italy, * \\ Dipartimento di Fisica Nucleare e Teorica, Università di Pavia, \\ and INFN, Sezione di Pavia, Pavia, Italy** \\ and \\ Theory Division, CERN, Geneva, Switzerland.
}

\begin{abstract}
We first show that nonrelativistic quantum mechanics formulated at imaginary- $\hbar$ can formally be viewed as the Fokker-Planck description of a frictionless brownian motion, which occurs (in general) in an absorbing medium. We next offer a new formulation of quantum mechanics, which is basically the Langevin treatment of this brownian motion. Explicitly, we derive a noiseaverage representation for the transition probability $W\left(X^{\prime \prime}, t^{\prime \prime} \mid X^{\prime}, t^{\prime}\right)$, in terms of the solutions to a Langevin equation with a gaussian white-noise. Upon analytic continuation back to real$\hbar, W\left(X^{\prime \prime}, t^{\prime \prime} \mid X^{\prime}, t^{\prime}\right)$ becomes the propagator of the original Schrödinger equation. Our approach allows for a straightforward application to quantum dynamical problems of the mathematical techniques of classical stochastic processes. Moreover, computer simulations of quantum mechanical systems can be carried out by using numerical programs based on the Langevin dynamics.
\end{abstract}

* Present address.

** Permanent address.

IC $/ 89 / 40$

CERN-TH.5360/89

March 1989 
Analogies between quantum mechanics and stochastic diffusion processes have been discovered [1] since the beginning of the quantum theory, and subsequently investigated by many authors [2]. Yet, the peculiar nature of quantum fluctuations implies that it is impossible to view quantum mechanics as a classical stochastic theory [3]. Still, the exciting possibility of handling and solving quantum dynamical problems by exploiting analytical and numerical (computer assisted) techniques of classical stochastic processes makes any work on this program well worth the effort.

A natural strategy relies upon the fact that quantum fluctuations become classical statistical fluctuations once quantum dynamics is formulated either at imaginary-time ( $s \equiv i t)$ or at imaginary- $\hbar(\hbar \equiv-i \lambda)$.

Consider a point particle $S$ (mass $m$ and no spin) with configuration space $\mathbb{R}^{N}$ (this is the dynamical system dealt with throughout this Letter).

Suppose first that $\mathcal{S}$ is described by the lagrangian

$$
L_{*}(X, \dot{X})=\frac{1}{2} m \dot{X}_{i} \dot{X}_{i}-\Phi_{*}(X)
$$

with $\Phi_{*}(X)$ time-independent. As long as the corresponding quantum hamiltonian has a finiteenergy normalizable ground-state, the imaginary-time Schrödinger equation can be written as [4]

$$
\frac{\partial}{\partial s} W_{*}(X, s)=\left[\frac{\hbar}{2 m} \frac{\partial^{2}}{\partial X^{2}}+\frac{1}{2 m} \frac{\partial^{2} \theta}{\partial X^{2}}-\frac{1}{2 m \hbar}\left(\frac{\partial \theta}{\partial X_{i}}\right)^{2}\right] W_{*}(X, s)
$$

where $\theta(X)$ is the solution of the Riccati equation

$$
\frac{1}{2 m}\left(\frac{\partial \theta}{\partial X_{i}}\right)^{2}-\frac{\hbar}{2 m} \frac{\partial^{2} \theta}{\partial X^{2}}-\Phi_{*}(X)=0
$$

By further introducing the new function $P(X, s) \equiv \exp [-\theta(X) / \hbar] \cdot W_{*}(X, s)$, Eq.(2) becomes

$$
\frac{\partial}{\partial s} P(X, s)=\left[\frac{\hbar}{2 m} \frac{\partial^{2}}{\partial X^{2}}-\frac{\partial}{\partial X_{i}}\left(-\frac{1}{m} \frac{\partial \theta}{\partial X_{i}}\right)\right] P(X, s)
$$

which is a standard Fokker-Planck equation. As a result, $P(X, s)$ can be regarded as the probability density of a classical stochastic diffusion process in $\mathbf{R}^{N}$, with diffusion constant $\hbar / 2 m$ and 
drift velocity $-(1 / m) \nabla \theta(X)$. Thus - according to this specific picture - quantum mechanics at imaginary-time describes a frictionless brownian motion with conserved particle number, which satisfies the potential conditions [5]. As a matter of fact, the stochastic correlation functions yield the quantum mechanical Green's functions [6] at imaginary-time and the equal-time statistical averages give (as $s \rightarrow \infty$ ) the imaginary-time quantum expectations in the ground-state. All this is well known [7], along with the supersymmetric structure of the partition function [8].

Most important for us, there exists an alternative connection - more straightforward [9] and more general [10] even if much less known - between quantum theory at imaginary - $\hbar$ (equivalently imaginary-time) and classical stochastic processes.

Suppose now that $S$ is described by the more general lagrangian

$$
L(X, \dot{X}, t)=\frac{1}{2} m \dot{X}_{i} \dot{X}_{i}+\Omega_{i}(X, t) \dot{X}_{i}-\Phi(X, t)
$$

where the potentials $\Phi(X, t), \Omega(X, t)$ are assumed to be even and odd, respectively, under timereversal, thereby implying time-reversal invariance of the equations of motion. (Still more general situations will be considered elsewhere.) Correspondingly, the Schrödinger equation at imaginary $-\hbar(\hbar \equiv-i \lambda)$ reads

$$
\begin{gathered}
\frac{\partial}{\partial t} W(X, t)=\left\{\frac{\lambda}{2 m} \frac{\partial^{2}}{\partial X^{2}}-\frac{\partial}{\partial X_{i}}\left(-\frac{1}{m} \Omega_{i}(X, t)\right)\right. \\
\left.-\frac{1}{\lambda}\left[\frac{\lambda}{2 m} \frac{\partial \Omega_{i}}{\partial X_{i}}-\frac{1}{2 m} \Omega_{i}(X, t) \Omega_{i}(X, t)-\Phi(X, t)\right]\right\} W(X, t) .
\end{gathered}
$$

Setting further

$$
\begin{gathered}
V_{i}(X, t) W(X, t) \equiv-\frac{1}{m} \Omega_{i}(X, t) W(X, t)-\frac{\lambda}{2 m} \frac{\partial W}{\partial X_{i}} \\
\mathcal{D}(X, t) \equiv \frac{1}{\lambda}\left[\frac{\lambda}{2 m} \frac{\partial \Omega_{i}}{\partial X_{i}}-\frac{1}{2 m} \Omega_{i}(X, t) \Omega_{i}(X, t)-\Phi(X, t)\right]
\end{gathered}
$$

Eq.(6) takes the form

$$
\frac{\partial}{\partial t} W(X, t)=-\frac{\partial}{\partial X_{i}}\left[V_{i}(X, t) W(X, t)\right]-\mathcal{D}(X, t) W(X, t)
$$

We stress that we are going to work throughout at imaginary- $\hbar$ (real $\lambda$ ). Our point of view is to interpret [11]: $W(X, t)$ as the probability density to find $S$ in $X$ at time $t$. Accordingly, the 
transition probability $W\left(X^{\prime \prime}, t^{\prime \prime} \mid X^{\prime}, t^{\prime}\right)$ - propagator of Eq. (6) - enjoys the generalized WienerOnsager-Machlup [12] path-integral representation

$$
\begin{gathered}
W\left(X^{\prime \prime}, t^{\prime \prime} \mid X^{\prime}, t^{\prime}\right) \sim \int \mathcal{D} X \delta\left(X^{\prime \prime}-X\left(t^{\prime \prime}\right)\right) \delta\left(X^{\prime}-X\left(t^{\prime}\right)\right) \\
\exp \left\{-(1 / \lambda) \int_{t^{\prime}}^{t^{\prime \prime}} d t\left[\frac{1}{2} m \dot{X}_{i} \dot{X}_{i}+\Omega_{i}(X, t) \dot{X}_{i}-\Phi(X, t)\right]\right\}
\end{gathered}
$$

where the exponential on the r.h.s. of Eq.(9) is a (nonnormalized) probability distribution $P[X(t)]$ on the space $C\left(X^{\prime}, t^{\prime} ; X^{\prime \prime}, t^{\prime \prime}\right)$ of all continuous paths $X(t)$ in $\mathbb{R}^{N}$ with fixed end-points $X\left(t^{\prime}\right)=$ $X^{\prime}, X\left(t^{\prime \prime}\right)=X^{\prime \prime}$. Now, a glance at Eqs.(7a), (8) shows that the imaginary- $\hbar$ quantum dynamics corresponding to Lagrangian (5) can formally be viewed as the Fokker-Planck description in $\mathbf{R}^{N}$ of a frictionless brownian motion with diffusion constant $\lambda / 2 m$ and drift velocity $-(1 / m) \Omega(X, t)$. Moreover, this stochastic diffusion process - performed by $\mathcal{S}$-occurs in a medium where particles can be absorbed and emitted, with killing rate given by $\mathcal{D}(X, t)$ in Eq.(7b). Notice that the potential conditions are no longer obeyed (in general).

We present a new formulation of quantum mechanics based on this scenario - it is formally the Langevin description of the brownian motion in question. Specifically, we offer a noiseaverage representation for the transition probability $W\left(X^{\prime \prime}, t^{\prime \prime} \mid X^{\prime}, t^{\prime}\right)$, in terms of the solutions to a Langevin equation with a gaussian white-noise. Obviously, the analytic continuation of $W\left(X^{\prime \prime}, t^{\prime \prime} \mid X^{\prime}, t^{\prime}\right)$ back to real- $\hbar$ yields the propagator of the original Schrödinger equation [13].

Actually, we want to formulate quantum dynamics at imaginary- $\hbar$ as a classical stochastic diffusion process $\xi(t)$ in $\mathbb{R}^{N}$ defined by the probability density $W(X, t)$ and the transition probability $W\left(X^{\prime \prime}, t^{\prime \prime} \mid X^{\prime}, t^{\prime}\right)(\xi(t)$ describes the random position of $\mathcal{S}$ in this picture).

As a preliminary step, consider the case where the potentials obey the condition

$$
\frac{\lambda}{2 m} \frac{\partial \Omega_{i}}{\partial X_{i}}-\frac{1}{2 m} \Omega_{i}(X, t) \Omega_{i}(X, t)-\Phi(X, t)=0
$$

so that the number of particles is now conserved, and Eq.(6) becomes

$$
\frac{\partial}{\partial t} W_{0}(X, t)=\left[\frac{\lambda}{2 m} \frac{\partial^{2}}{\partial X^{2}}-\frac{\partial}{\partial X_{i}}\left(-\frac{1}{m} \Omega_{i}(X, t)\right)\right] W_{0}(X, t)
$$


which is a standard Fokker-Planck equation. Next, it follows from the general theory of stochastic processes [5],[14] that $\xi(t)$ satisfies the Langevin equation

$$
\frac{d}{d t} \xi_{i}(t)=-\frac{1}{m} \Omega_{i}(\xi(t), t)+\eta_{i}(t)
$$

where $\eta(t)$ is a gaussian white noise defined as

$$
\begin{aligned}
& \left\langle\eta_{i}(t)\right\rangle_{\eta}=0 \\
& \left\langle\eta_{i}(t) \eta_{j}\left(t^{\prime}\right)\right\rangle_{\eta}=\frac{\lambda}{m} \delta_{i j} \delta\left(t-t^{\prime}\right) .
\end{aligned}
$$

Assuming that $\eta(t)$ is odd under $t \rightarrow-t$, Eq.(12) is manifestly time-reversal invariant, so that $\xi(t)$ indeed describes a frictionless brownian motion [15]. Now, the transition probability between $\left(X^{\prime}, t^{\prime}\right)$ and $\left(X^{\prime \prime}, t^{\prime \prime}\right)\left(t^{\prime \prime} \geq t^{\prime}\right)$ in the case of a single, arbitrary noise realization $\eta(t)$ is obviously

$$
W_{0}\left(X^{\prime \prime}, t^{\prime \prime} \mid X^{\prime}, t^{\prime}\right)[\eta(t)]=\delta\left(X^{\prime \prime}-\xi\left(\eta\left(t^{\prime \prime}\right), t^{\prime \prime} ; X^{\prime}, t^{\prime}\right)\right)
$$

where $\xi\left(\eta(t), t ; X^{\prime}, t^{\prime}\right)$ denotes the solution of Eq.(12) with initial condition $\xi=X^{\prime}$ at $t=t^{\prime}$. Correspondingly, the transition probability between $\left(X^{\prime}, t^{\prime}\right)$ and $\left(X^{\prime \prime}, t^{\prime \prime}\right)$ is

$$
W_{0}\left(X^{\prime \prime}, t^{\prime \prime} \mid X^{\prime}, t^{\prime}\right)=\left\langle W_{0}\left(X^{\prime \prime}, t^{\prime \prime} \mid X^{\prime}, t^{\prime}\right)[\eta(t)]\right\rangle_{\eta}=\left\langle\delta\left(X^{\prime \prime}-\xi\left(\eta\left(t^{\prime \prime}\right), t^{\prime \prime} ; X^{\prime}, t^{\prime}\right)\right)\right\rangle_{\eta}
$$

which is the way the propagator of the Fokker-Planck equation arises in the context of Langevin dynamics.

Unfortunately, condition (10) turns out to be a very strong limitation for realistic situations, and so we proceed to relax it (thus the potentials will be free of any constraint from now on). Manifestly, absorption and emission processes are now operative, but the stochastic dynamics of $S$ is still described by Eq.(12). Suppose that $S$ is in $X^{\prime}$ at time $t^{\prime}$, and again consider first the case of dynamical behaviour in the presence of a single noise sample $\eta(t)$. Correspondingly, $\mathcal{S}$ will start following the path $\xi\left(\eta(t), t ; X^{\prime}, t^{\prime}\right)$ given by Eq.(12). Now, elementary probabilistic considerations tell that the probability for $S$ to survive this path - without being absorbed - during the time interval $t^{\prime} \leq t \leq t^{\prime \prime}$ is

$$
\exp \left\{-\int_{t^{\prime}}^{t^{\prime \prime}} d t \mathcal{D}\left(\xi\left(\eta(t), t ; X^{\prime}, t^{\prime}\right), t\right)\right\}
$$


Recalling Eq.(14) for the motion without absorption, the transition probability between $\left(X^{\prime}, t^{\prime}\right)$ and $\left(X^{\prime \prime}, t^{\prime \prime}\right)$ in the presence of the single noise realization $\eta(t)$ is then

$$
\begin{gathered}
W\left(X^{\prime \prime}, t^{\prime \prime} \mid X^{\prime}, t^{\prime}\right)[\eta(t)]=\delta\left(X^{\prime \prime}-\xi\left(\eta\left(t^{\prime \prime}\right), t^{\prime \prime} ; X^{\prime}, t^{\prime}\right)\right) \\
\exp \left\{-\int_{t^{\prime}}^{t^{\prime \prime}} d t \mathcal{D}\left(\xi\left(\eta(t), t ; X^{\prime} t^{\prime}\right), t\right)\right\}
\end{gathered}
$$

We conclude that the corresponding transition probability is given by

$$
\begin{gathered}
W\left(X^{\prime \prime}, t^{\prime \prime} \mid X^{\prime}, t^{\prime}\right)=\left\langle W\left(X^{\prime \prime}, t^{\prime \prime} \mid X^{\prime}, t^{\prime}\right)[\eta(t)]\right\rangle_{\eta}=\left\langle\delta\left(X^{\prime \prime}-\xi\left(\eta\left(t^{\prime \prime}\right), t^{\prime \prime} ; X^{\prime}, t^{\prime}\right)\right)\right. \\
\left.\exp \left\{-\left.(1 / \lambda) \int_{t^{\prime}}^{t^{\prime \prime}} d t\left[\frac{\lambda}{2 m} \frac{\partial \Omega_{i}}{\partial X_{i}}-\frac{1}{2 m} \Omega_{i}(X, t) \Omega_{i}(X, t)-\Phi(X, t)\right]\right|_{X=\xi\left(\eta(t), t ; X^{\prime} t^{\prime}\right)}\right\}\right\rangle_{\eta}
\end{gathered}
$$

which is our final result, namely the noise-average representation of the quantum mechanical propagator at imaginary- $\hbar$ (corresponding to lagrangian (5)).

As is well known, only trajectories $X(t)$ of $C\left(X^{\prime}, t^{\prime} ; X^{\prime \prime}, t^{\prime \prime}\right)$ which are Hölder functions of class one-half [16] actually contribute to the path-integral (9). Amusingly, their probability distribution $P[X(t)]$ can be expressed as a noise average involving $W\left(X^{\prime \prime}, t^{\prime \prime} \mid X^{\prime}, t^{\prime}\right)[\eta(t)]$

$$
P[X(t)]=\left\langle\delta\left[X(t)-\xi\left(\eta(t), t ; X^{\prime}, t^{\prime}\right)\right] W\left(X^{\prime \prime}, t^{\prime \prime} \mid X^{\prime}, t^{\prime}\right)[\eta(t)]\right\rangle_{\eta}
$$

as it can be shown by standard manipulations.

It should be observed that essentially the same picture as discussed above can alternatively be obtained by performing a double analytic continuation - instead of in $\hbar-$ in $t$ and in the potentials

$$
\begin{gathered}
t \longrightarrow s \equiv i t \\
\Omega_{i}(X, t) \longrightarrow \tilde{\Omega}_{i}(X, s) \equiv i \Omega_{i}(X, t) \\
\Phi(X, t) \longrightarrow \tilde{\Phi}(X, s) \equiv \Phi(X, t)
\end{gathered}
$$

which leads to a noise-average representation of the quantum mechanical propagator at imaginarytime quite similar to Eq.(18). Remarkably enough, in this case the stochastic interpretation of Eq.(2) 
can be recovered as the particular way of fulfilling the analog of Eq.(10) in a manner consistent with the potential conditions. Explicitly

$$
\begin{gathered}
\tilde{\Omega}_{i}(X)=-\frac{\partial \theta}{\partial X_{i}} \\
\tilde{\Phi}(X)=\frac{1}{2 m}\left(\frac{\partial \theta}{\partial X_{i}}\right)^{2}-\frac{\hbar}{2 m} \frac{\partial^{2} \theta}{\partial X^{2}}
\end{gathered}
$$

which make the imaginary-time Schrödinger equation stemming from lagrangian (5) coincide with Eq.(4).

We have outlined a new approach to nonrelativistic quantum mechanics, motivated by the analogy with macroscopic brownian motion - see Table - and based on a stochastic differential equation in physical time. A crucial point should be stressed. Contrary to the case of Nelson's stochastic mechanics [17], the drift velocity in the Langevin equation (12) depends only on the classical potentials - not on the quantum mechanical wave function as in stochastic mechanics [18]. This fact implies that explicit quantum mechanical problems can be formulated and solved by using standard methods of classical stochastic processes. Moreover, computer simulations of quantum dynamical systems can be performed by employing numerical programs based on the Langevin dynamics [14] - they play a rôle alternative to the Monte Carlo technique defined in the context of the path-integral approach at imaginary-time (or imaginary- $\hbar$ ) [19]. We would like to anticipate that the knowledge of the classical solution of a specific dynamical problem brings about a drastic simplification in the explicit evaluation of r.h.s. of Eq.(18) - the integral becomes trivial! This and various other aspects of the strategy presented here will be discussed elsewhere.

\section{ACKNOWLEDGEMENTS.}

The author would like to thank Professor Abdus Salam, the International Atomic Energy Agency and UNESCO for hospitality at the International Centre for Theoretical Physics, Trieste. It is a pleasure for him to thank F. Guerra, G. Jona-Lasinio and G. Parisi for conversations and encouragement. 


\section{TABLE}

QUANTUM MECHANICS

CONVENTIONAL FORMULATION

Density of probability amplitude as solution of the Schrödinger equation

PATH-INTEGRAL FORMULATION (FEYNMAN)

Transition amplitude (propagator) as sum over random paths.

PRESENT FORMULATION

Transition amplitude (propagator) (analytically continued to $\hbar \equiv-i \lambda$ ) as average over a white-noise
BROWNIAN MOTION

FOKKER-PLANCK FORMULATION

Probability density as solution of the Fokker-Planck equation.

PATH-INTEGRAL FORMULATION (WIENER,ONSAGER,MACHLUP)

Transition probability (propagator) as sum over random paths.

\section{LANGEVIN FORMULATION}

Transition probability ( propagator) as average over a white-noise

Correspondence between the formulations of quantum mechanics and of macroscopic brownian motion. 


\section{REFERENCES AND FOOTNOTES}

[1] E. Schrödinger, Berl. Sitzber 144 (1931); Ann. Inst. H. Poincaré 2, 269 (1932).

R. Fürth, Z. Phys. 81, 143 (1933).

I. Fenyes, Z. Phys. 132, 81 (1952).

[2] See ref. [7],[17] and papers quoted therein.

[3] Superficially, Nelson's stochastic mechanics (see ref. [17]) may appear as a counterexample to this statement. This is however not the case, since the concept of wave packet reduction must be incorporated in stochastic mechanics in order to ensure consistency with quantum mechanics. See P. Blanchard, S. Golin and M. Serva, Phys. Rev. D34, 3732 (1986).

[4] We have performed the redefinition $W_{*}(X, t) \rightarrow e^{-E_{0} t / h} W_{*}(X, t)$.

[5] R.L. Stratonovich, Topics in the Theory of Random Noise, Vol. 1 (Gordon and Breach, New York, 1963).

[6] Namely, the expectation value in the ground-state of time-ordered products of position operators in the Heisenberg picture.

[7] N.G. Van Kampen, J: Stat. Phys. 17, 71 (1977)

T. Schneider, M. Zannetti and R. Badii, Phys. Rev. B31, 2941 (1985).

H. Risken, The Fokker-Planck Equation (Springer, Berlin, 1984).

G. Parisi, Statistical Field Theory (Addision-Wesley, New York, 1988).

[8] E. Gozzi, Phys. Lett. 129B, 432 (1983).

[9] No equation like Eq.(3) has to be solved as a preliminary step.

[10] It applies to a general, time-dependent hamiltonian quadratic in the momentum.

[11] This interpretation - even if not compulsory - looks natural and physically consistent. We would like to stress that all the results of this Letter can also be derived by formal manipulations starting from Eq.(9) without any assumption about the meaning of $W(X, t)$. 
[12] I.M. Gel'fand and A.M. Yaglom, J. Math. Phys. 1, 48 (1960)

R. Graham, Z. Phys. B26, 281 (1977).

[13] A preliminary account of this approach has been presented at the Conference "Feynman's Quantum Mechanics 40 years after its proposal" (Naples, June 16-18, 1988).

See: M. Roncadelli, Nuovo Cim. 11D, 73 (1989).

[14] H. Risken, The Fokker-Planck Equation (Springer, Berlin 1984).

[15] I. Prigogine, Irreversibility in Mechanics and Thermodynamics, preprint (unpublished). The situation is formally identical to what happens in Nelson's stochastic mechanics (see Ref. [17]).

[16] H.P. McKean, Stochastic Integrals (Academic Press, New York, 1969).

[17] E. Nelson, Phys. Rev. 150, 1079 (1966).

G.C. Ghirardi, C. Omero, A. Rimini and T. Weber, Riv. Nuovo Cimento, Vol. 1, N. 3 (1978).

F. Guerra, Phys. Rep. 77, 263 (1981).

E. Nelson, Quantum Fluctuations (Princeton University Press, Princeton, 1984).

[18] Actually, the Langevin equation of Nelson's stochastic mechanics can explicitly be written down only after the wave function is known, so that Nelson's approach does not permit (in general) to solve quantum mechanical problems by using techniques of classical stochastic processes.

[19] M. Creutz and B. Freedman, Am. Phys. (N.Y.) 132, 427 (1981). 